\title{
Structure and Morphology of Gold Nanoparticles in Solution Studied by TEM, SAXS and UV-Vis
}

\author{
M. MuraWska ${ }^{a}$, A. SkrzypCzAK ${ }^{b}$, M. KOzAK $^{a *}$ \\ ${ }^{a}$ Department of Macromolecular Physics, Faculty of Physics, A. Mickiewicz University \\ Umultowska 85, 61-614 Poznań, Poland
}

${ }^{b}$ Faculty of Chemical Technology, Poznań University of Technology, Piotrowo 3, 60-965 Poznań, Poland

Gold nanoparticles have a great number of applications, among others in material sciences, biology and medicine. A method for the synthesis of gold nanoparticles in solution with the use of gemini surfactant was proposed and the nanoparticles obtained were subjected to thorough characterisation. The method proposed is a modification of the Turkevich method, based on reduction of tetrachloroauric acid in the presence of trisodium citrate and a dicationic (gemini) surfactant - 1,1'-(1,4-butan) bis(3-dodecyloxymethylimidazolium) di-propionate. Morphology and size distribution of gold nanoparticles obtained were examined by the transmission electron microscopy (TEM), UV-Vis spectroscopy and small angle scattering of synchrotron radiation (SAXS). The plasmon resonance of the nanoparticles obtained was observed in the wavelength range corresponding to the presence of gold nanoparticles with sizes ranging from 5 to $100 \mathrm{~nm}$. TEM images confirmed that the spherical shape of nanoparticles was dominated in reference solutions prepared of sodium citrate and tetrachloroauric acid. In the solutions prepared with addition of gemini surfactant, the gold nanoparticles of triangular morphology were observed.

PACS: 61.05.cf, 61.46.Hk, 68.37.Lp, 33.20.Kf, 81.16.Be

\section{Introduction}

Gold nanoparticles (GNP) have enjoyed increasing interest because of a wide range of their potential applications in such fields as: medicine, biotechnology, catalysis, preparation of nanocomposites, nanosensing and electronics. The potential applications of GNP depend on their size and morphology. Especially the potential application of GNP in nanosensing is strongly connected with controlling of their size and morphology by the proper choice of preparation conditions [1-3].

There are a few general methods for the synthesis of gold nanoparticles. The most popular are chemical or photochemical reduction of gold precursor [3-8] and biosynthesis $[9,10]$. Chemical methods are the most efficient and convenient, because they require a relatively simple equipment and nanoparticle solutions obtained are relatively stabile and monodisperse.

The chemical reduction of metal ions is the most common method of GNP synthesis. This method consists in precipitation of gold nanoparticles from a solution of a dissolved gold precursor under the influence of a reducing agent $[4,5]$. A gold precursor usually is tetrachloroauric acid $\left(\mathrm{HAuCl}_{4}\right)$ while a reducing agent could be sodium citrate [4,5], sodium boron hydride [11], block copolymers [12] or ascorbic acid. The reduction of gold precursor with sodium citrate is the simplest and the most reliable method of obtaining a solution of monodisperse gold nanoparticles. This method was proposed by Turkevich [4] and is used as the model reaction in the

\footnotetext{
* e-mail: mkozak@amu.edu.pl
}

present study. Unfortunately the Turkevich method can only produce particles up to about $40-50 \mathrm{~nm}$ in size. Highly monodispersed large-diameter GNPs can be synthesized from $\mathrm{HAuCl}_{4}$ by hydroquinone (HQ) reduction [13]. This method provides a greater size range of GNPs (50-250 nm) and very good shape dispersion.

Another chemical method of preparation of gold nanoparticles is a method discovered by the Burst and coworkers in the early 1990s [14]. This method allows to prepare gold nanoparticles in the two phase system (toluene/water). The gold precursor $\left(\mathrm{HAuCl}_{4}\right)$ is transferred from aqueous solution to toluene using tetraoctylammonium bromide (TOAB) as the phase-transfer reagent and reduced by sodium borohydride in the presence of an alkanethiol (dodecanethiol, $\mathrm{C}_{12} \mathrm{H}_{23} \mathrm{SH}$ ). The colloidal solutions of nanoparticles obtained are very stable and have a size 1-3 nm [14].

In the present work the synthesis of gold nanoparticles in solution in the presence of a gemini surfactant was studied. The gemini surfactant (also known as dimeric or dicationic surfactant) is made of two amphiphilic head groups connected at the same level or close to the head group by a flexible or rigid spacer group [15-17]. Ionic surfactants were used as templates and stabilizers in the preparation of metallic nanoparticles [18]. Gemini surfactants as compared to monomeric surfactants are characterized by 1 to 2 orders of magnitude lower critical concentration of micellisation CMC, which allows to use much smaller amounts of a surfactant in the process of GNP synthesis.

The aim of this work was a development of the synthesis method of gold nanoparticles in the presence 
of dicationic (gemini) surfactant: 1,1'-(1,4-butan)bis(3dodecyloxymethyl-imidazolium) di-propionate (12-4-12). The nanoparticles obtained were characterised by the transmission electron microscopy (TEM), UV-Vis spectroscopy and small angle scattering of synchrotron radiation (SAXS).

\section{Experimental details}

\subsection{Materials}

Tetrachloroauric acid $\left(\mathrm{HAuCl}_{4}\right)$ and sodium citrate were obtained from Sigma-Aldrich. The gemini surfactant: 1,1'-(1,4-butan)bis $(3-$ dodecyloxymethylimidazolium) di-propionate (Fig. 1) was prepared as described in [19].

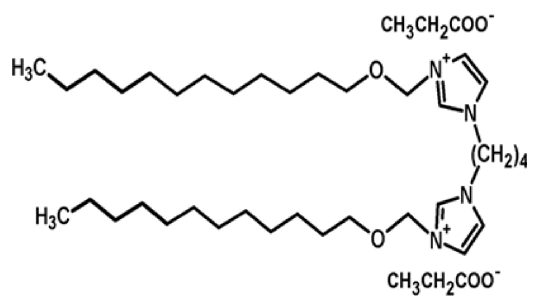

Fig. 1. Chemical structure of the gemini surfactant 1,1'-(1,4-butan $)^{\prime}$ (3-dodecyl-oxymethylimidazolium) dipropionate, used for the synthesis of gold nanoparticles.

\subsection{Nanoparticle synthesis}

The GNP synthesis was based on the Turkevich method [4]. $\mathrm{HAuCl}_{4}$ was a gold precursor and trisodium citrate was a reducing agent. In accordance with the standard procedure the reaction temperature was $75^{\circ} \mathrm{C}$ $[4,5]$. The reference samples were obtained by addition of trisodium citrate solution to a water solution of $\mathrm{HAuCl}_{4}$ in $75^{\circ} \mathrm{C}$ under continuous stirring. The final concentration of the ingredients was $0.25 \mathrm{mmol} / \mathrm{L}$ of $\mathrm{HAuCl}_{4}$ and $2.5 \mathrm{mmol} / \mathrm{L}$ of trisodium citrate. The reference sample finally got deep ruby-red in colour. The samples synthesized in the presence of a gemini surfactant were prepared by using the same method. The surfactant 1,1'-(1,4-butan) bis(3-dodecyloxymethylimidazolium) dipropionate (GC4C12prop) was dissolved in millipore water and added to the gold precursor solution before reduction and preheated to the reaction temperature. The next step was the addition of citrate solution under continuous stirring. The gold nanoparticles were prepared at different gemini surfactant concentrations and at various amounts of citrate solution. All synthesis steps were carried out in test-tubes immersed in a temperaturecontrolled water bath.

\subsection{Methods \\ 2.3.1. UV-vis spectrophotometry}

The absorption measurement in $\mathrm{UV}-\mathrm{V}$ is range were used for the independent examination of the size and shape of nanoparticles [20]. UV-visible spectra of nanoparticle solutions prepared were taken using a
JASCO V-650 spectrophotometer, over the wavelength range $350-800 \mathrm{~nm}$. The process of formation of nanoparticles was controlled by observation of the absorption maximum at around $\approx 530 \mathrm{~nm}$.

\subsection{2. $T E M$}

The morphology and size of nanoparticles obtained were examined by transmission electron microscopy (TEM). The GNPs solutions were dropped on standard carbon-coated copper grids (200-mesh) and air dried for about $2 \mathrm{~h}$. The TEM images were obtained using a JEM 1200 EX II (Jeol Co., Japan) transmission electron microscope, operating at $80 \mathrm{kV}$. Size distribution of the nanoparticles was determined on the basis of TEM images with the use of the Image $\mathrm{J}$ program (http://rsbweb.nih.gov/ij/).

\subsection{3. $S A X S$}

Preliminary small angle X-ray scattering patterns were collected using Nano STAR (Bruker-AXS) with pin-hole collimation and a HiSTAR detector, mounted on an Xray tube with copper anode and equipped with crossed Göbel mirrors. The sample-to-detector distance was 650 $\mathrm{mm}$. The intensities were recorded within the range of $0.15 \mathrm{~nm}^{-1}<s<3.5 \mathrm{~nm}^{-1}(s=4 \pi \sin \theta / \lambda$, where $2 \theta$ is the scattering angle and $\lambda$ is the wavelength).

The synchrotron SAXS data were collected on the Beam Line I911-4 [21, 22] at the MAXII storage ring of the MAX-Lab (Lund, Sweden) using the synchrotron radiation $(\lambda=0.091 \mathrm{~nm})$ and the MarCCD $165 \mathrm{~mm}$ detector. The GNP samples were injected into thermostated capillary sample holder and the measurements were performed at room temperature. The background scattering data for the buffer solution were recorded before and after data collection for GNP samples. The sample-to-detector distance was $1.9 \mathrm{~m}$, which leads to the scattering vector range $0.1 \mathrm{~nm}^{-}<s<3.55 \mathrm{~nm}^{-1}$. The detector s-axis was calibrated using silver behenate [23]. The data were normalized, corrected for detector response and integrated using SAXS data reduction software Bli711 [22]. The distance distribution function $p(r)$ was calculated from the experimental scattering also by indirect Fourier transformation and the program GNOM [24].

\section{Results and discussion}

TEM images confirmed the spherical shape of nanoparticles obtained by the modified Turkevich method (reduction of $\mathrm{HAuCl}_{4}$ solution with sodium citrate). The morphology of reference nanoparticles is presented in Figs. 2a. On the basis of the TEM images the size distribution of nanoparticles was calculated. The precise size distribution of nanoparticles is presented in Fig. 3a and the nanoparticles are characterised by diameters in the range $6-16.5 \mathrm{~nm}$. The average particle size was 9 $\mathrm{nm}$ for the solution of reference nanoparticles (without the addition of surfactant) (see Fig. 4). The nanoparticles obtained as a result of reduction of $\mathrm{HAuCl}_{4}$ by sodium citrate in the presence of the dimeric surfactant 1,1'-(1,4-butan)bis(3-dodecyloxymethylimidazolium) dipropionate are presented in Figs. $2 \mathrm{~b}-\mathrm{e}$ and $3 \mathrm{~b}-\mathrm{e}$. 

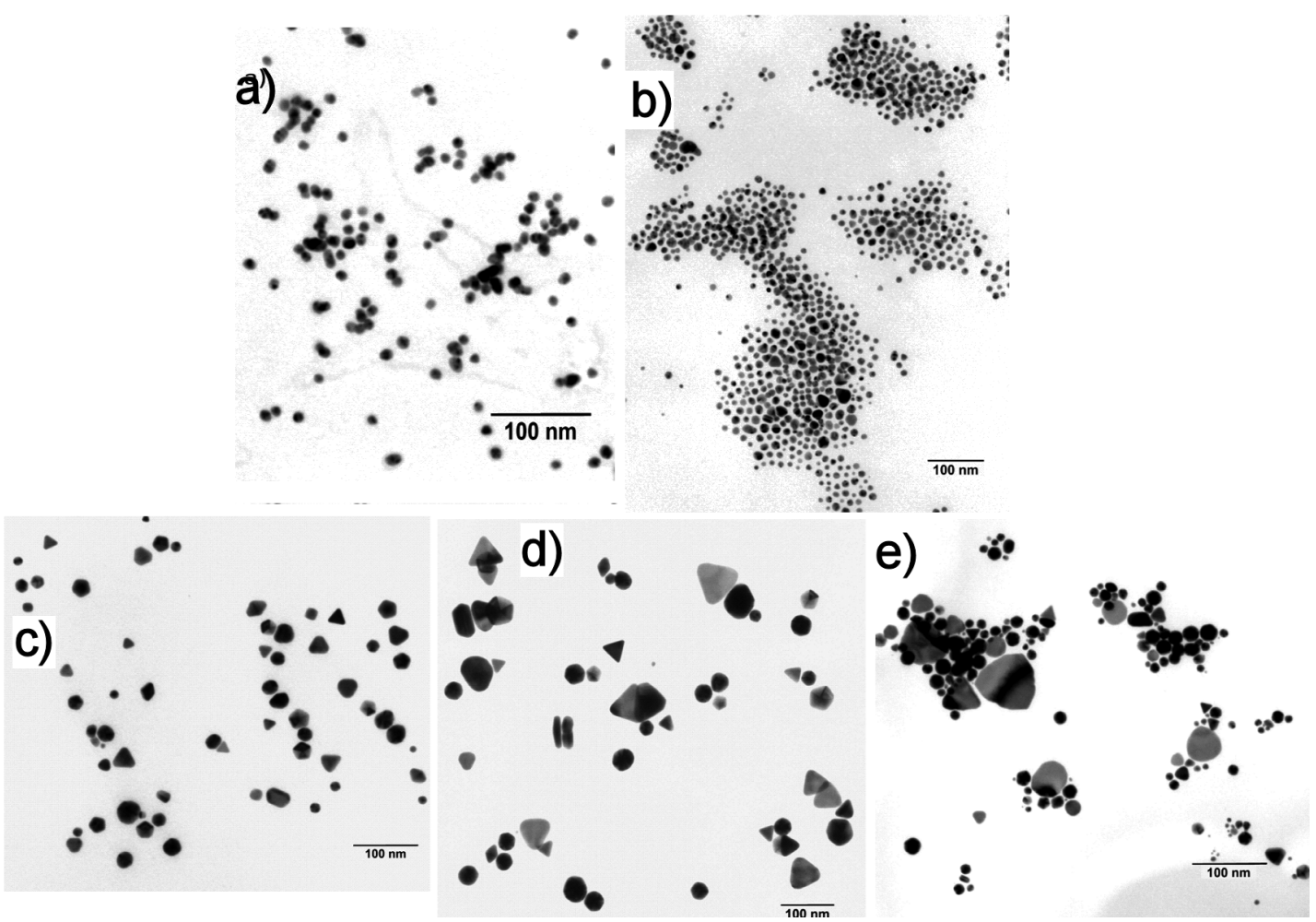

Fig. 2. TEM images of gold nanoparticles for: reference sample (without gemini surfactant) a); and samples prepared with: b) $0.0088 \%$ c) $0.0165 \%$ d) $0.035 \%$ and e) $0.07 \%$ gemini surfactant.

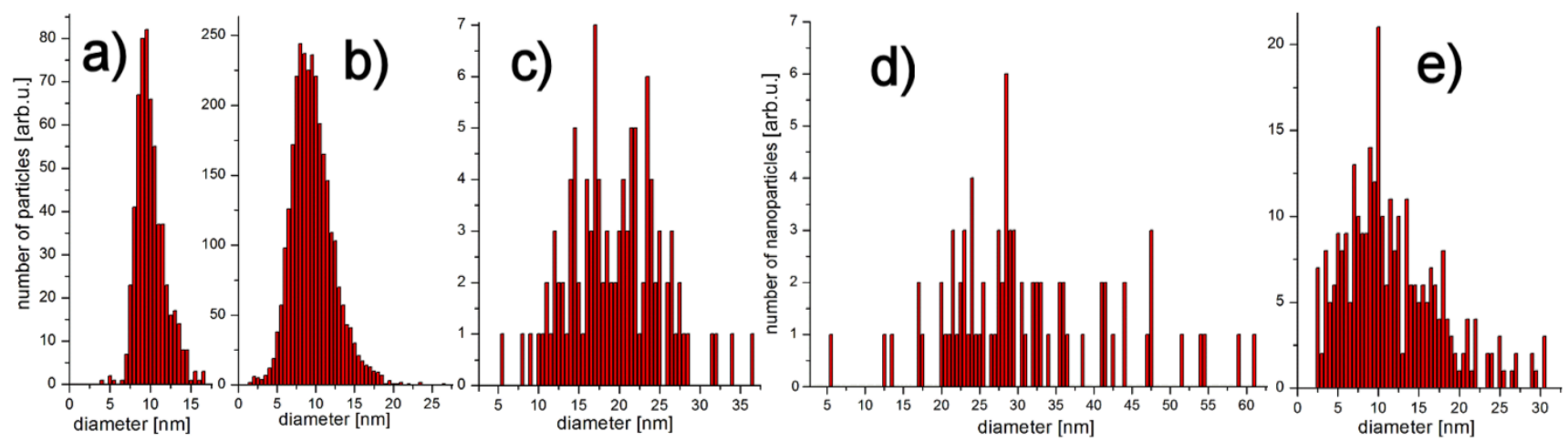

Fig. 3. Particle size distribution for: reference sample (without gemini surfactant) a); and samples prepared with: b) $0.0088 \%$ c) $0.0165 \%$ d) $0.035 \%$ and e) $0.07 \%$ gemini surfactant.

Even a small addition of a gemini surfactant initiates changes in the shape of nanoparticles and single tetrahedral particles can be observed. In the samples with concentration $0.0165 \%$ and $0.035 \%(\mathrm{w} / \mathrm{w})$ of dicationic surfactant several types of shapes are visible: spherical, tetrahedral, double tetrahedral pyramid, pentagonal pyramid and truncated tetrahedron (see Fig. $2 \mathrm{c}-\mathrm{d}$ ). The nanoparticles reached the maximum average size $(28.5$ $\mathrm{nm}$ ) for the surfactant concentration of $0.035 \%$ (Figs. 3d and 4 ). The greatest problem is quite high heterogeneity of shapes and sizes in samples obtained in the presence of a gemini surfactant. The size range calculated for these nanoparticles is from $5 \mathrm{~nm}$ to even $60 \mathrm{~nm}$ within one sample. An important feature of metallic nanoparticles is the enhancement of their optical properties under the influence of the light, known as the surface plasmon resonance.

The surface plasmon resonance is observed at a specific frequencies of incident light and depends on the particle size, shape, dielectric constant of metal and the medium around nanoparticles [25]. The typical frequency of plasmon resonance for spherical gold nanoparticles varies from $520 \mathrm{~nm}$ to $530 \mathrm{~nm}[25,26]$. The formation of $\mathrm{Au}$ NP was confirmed by UV-VIS spectroscopy at a wavelength close to $530 \mathrm{~nm}$. The reference solution of Au NP (without gemini surfactant) gave maximum absorbance 


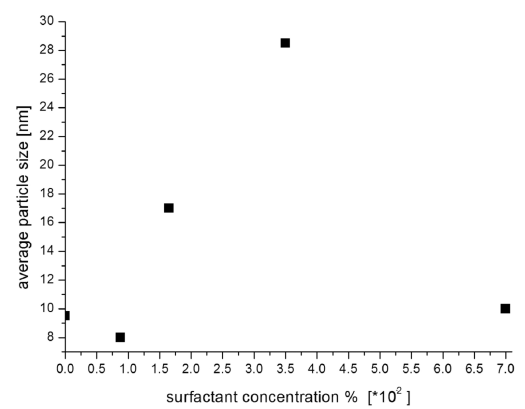

Fig. 4. Average particle size versus gemini surfactant concentration.

at $523 \mathrm{~nm}$ (Fig. 5). After the addition of a gemini surfactant the absorption peak is shifted towards longer wavelengths (Fig. 5). The absorbance peak for NP synthesized in the presence of $0.0088 \%$ gemini surfactant is observed at $540 \mathrm{~nm}$. The broadening of the peak shows substantial changes in the nanoparticles morphology. For $\mathrm{Au}$ nanoparticles obtained with $0.035 \%$ of gemini surfactant the largest broadening of the absorbance is visible. This indicates a high heterogeneity of size and shape within the sample.

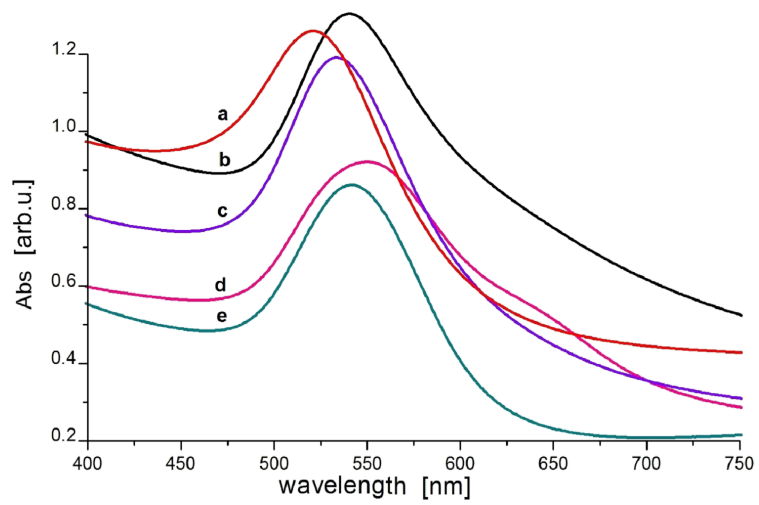

Fig. 5. UV-Vis spectra of gold nanoparticles a) reference sample (without gemini surfactant); and samples prepared with: b) $0.0088 \%$ c) $0.0165 \%$ d) $0.035 \%$ and e) $0.07 \%$ gemini surfactant.

Figure 6 presents the SAXS scattering curve for the reference solution of gold nanoparticles obtained using synchrotron radiation on the beam line I9-11 of MAXlab. The SAXS data are consistent with literature [5]. On the basis of the experimental curve the pair distance distribution $p(r)$ function (Fig. 7) was calculated. The maximum size of nanoparticles $D_{\max }=15 \mathrm{~nm}$ is consistent with the maximum size of nanoparticles obtained from the size distribution estimated on the basis of TEM data. The SAXS data for all samples of gold nanoparticles synthesized with and without the addition of a surfactant are shown in Fig. 6.

On the basis of fits of SAXS data to the Guinier equation [26] the gyration radii characterizing systems stud-

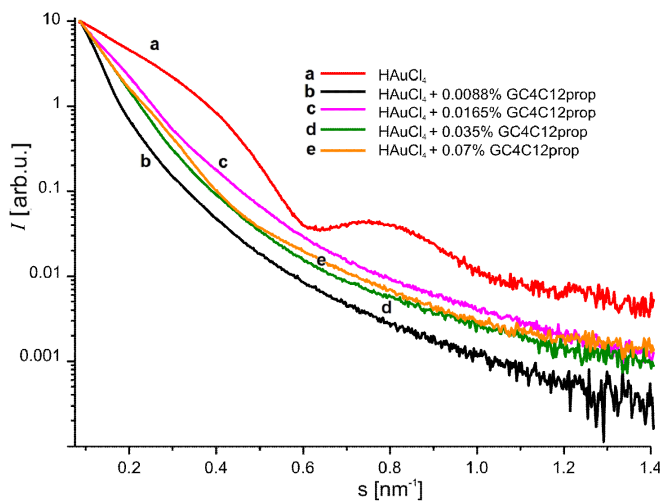

Fig. 6. SAXS scattering curves for the reference sample and gold nanoparticles prepared with the addition of surfactant.

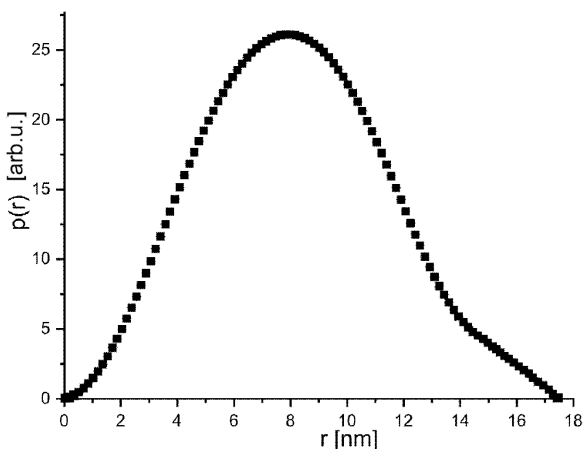

Fig. 7. The particle distance distribution function $\mathrm{p}(\mathrm{r})$ determined from SAXS curve for the gold nanoparticles without the addition of gemini surfactant.

ied were determined (Fig. 8). For the reference system the radius of gyration $R_{G}$ was $8.7 \mathrm{~nm}$. But for nanoparticles obtained in the presence of gemini surfactant radii of gyration, were higher reaching: $12.0 \mathrm{~nm}$ (0.0088\% GC4C12prop) $13.1 \mathrm{~nm}$ (0.0165\% GC4C12prop) $17.1 \mathrm{~nm}(0.035 \%$ GC4C12prop) and $13.9 \mathrm{~nm}(0.07 \%$ GC4C12prop). The observed changes of gyration radii

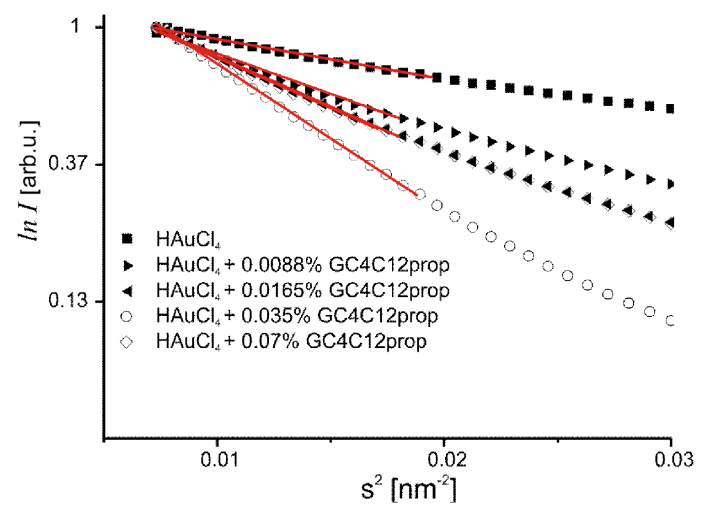

Fig. 8. Fits of SAXS data to the Guinier equation [26]. 
were connected with increasing surfactant concentration and are in good agreement with size distribution calculated from TEM. The shapes of the SAXS curves obtained for samples prepared in the presence of gemini surfactant exhibit high polydispersity of nanoparticles which is consistent with TEM results. Observed high polydispersity prevented the calculation of the $p(r)$ function for these samples. The morphology of nanoparticles obtained in presence of GC4C12prop were also similar to morphology of nanoparticles obtained by Bakshi et al. [27] using gemini surfactant trimethylene-1,3-bis (dodecyldimethylammonium bromide).

\section{Conclusions}

The phenomenon of plasmon resonance was observed for the GNP samples obtained in the wavelength range corresponding to the presence of gold nanoparticles with sizes from 5-100 nm [20]. TEM images confirmed the spherical morphology of the reference nanoparticles and showed the presence of gold nanoparticles of different shapes (e.g. tetrahedral and pentagonal) in solutions obtained in the presence of gemini surfactants. The shape and size of nanoparticles were found dependent on the concentration of the gemini surfactant. The average particle sizes increases with surfactant concentration (up to $0.035 \%$ ) reaching $28.5 \mathrm{~nm}$ for the surfactant concentration $0.035 \%$. However, for gemini surfactant concentrations of $0.0165 \%, 0.035 \%$ and $0.07 \%$ a large dispersion of particle sizes and a heterogeneity of their shapes was observed. Also the SAXS studies confirmed the sizes of nanoparticles obtained from analysis of TEM images. The future studies with other surfactants should improve homogeneity of shape and size of nanoparticles within one sample.

\section{Acknowledgments}

The study reported was carried out with financial support from the Ministry of Science and Higher Education (grant nr N N202 127237). The research (the data collection at Beam Line I911) leading to these results has received funding from the European Community's Seventh Framework Programme (FP7/2007-2013) under grant agreement no. 226716 .

\section{References}

[1] P. Yanez-Sedeno, J.M. Pingarron, Anal. Bioanal. Chem. 382, 884 (2005).

[2] S. Zeng, K.T. Yong, I. Roy, X.Q. Dinh, X. Yu, F. Luan, Plasmonics 6, 491 (2011).
[3] N. Zheng, J. Fan, G.D. Stucky, J. Am. Chem. Soc. 128, 6550 (2006).

[4] J. Turkevich, P.C. Stevenson, J. Hillier, Discuss Faraday Soc 11, 55 (1951).

[5] J. Polte, T.T. Ahner, F. Delissen, S. Sokolov, F. Emmerling, A.F. Thunemann, R. Kraehnert, J. Am. Chem. Soc. 132, 1296 (2010).

[6] T.C. Chiu, S.H. Chiou, M.M. Hsieh, Y.T. Chen, H.T Chang, J. Nanosci. Nanotechnol. 5, 2128 (2005).

[7] J. Perez-Juste, I. Pastoriza-Santos, L.M. Liz-Marzan, P. Mulvaney, Coord. Chem. Rev. 249, 1870 (2005).

[8] M. Hu, J.Y. Chen, Z.Y. Li, L. Au, G.V. Hartland, X.D. Li, M. Marquez, Y.N. Xia, Chem. Soc. Rev. 35, 1084 (2006)

[9] S.S. Shankar, A. Rai, B. Ankamwar, A. Singh, A. Ahmad, M. Sastry, Nature Materials 3, 482 (2004).

[10] M.A. Faramarzi, H. Forootanfar, Coll. Surf. BBiointerfaces 87, 23 (2011).

[11] T. Sakai, P. Alexandridis, Langmuir 20, 8426 (2004).

[12] J. Wagner, T.R. Tshikhudo, J.M. Kohler, Chem. Engineering J. 135, 104 (2008).

[13] S.D. Perrault, W.C.W. Chan, J. Am. Chem. Soc 131, 17042 (2009).

[14] M. Burst, M. Walker, D. Bethel, D.J. Schiffrin, J. Chem. Soc. Chem. Commun. 7, 801 (1994).

[15] R. Zana, Adv. Coll. Interface Sci. 97, 205 (2002).

[16] R. Zana, M. Benrraou, R. Rueff, Langmuir 7, 1072 (1991).

[17] F.M. Menger, C.A. Littau, J. Am. Chem. Soc. 113, 1451 (1991).

[18] T.S. Selvam, K.M. Chi, J. Nanopart. Res. 13, 1769 (2011).

[19] Z. Pietralik, M. Taube, A. Skrzypczak, M. Kozak, Acta Phys. Polon. A 117, 311 (2010).

[20] D.E. Mustafa, T. Yang, Z. Xuan, S. Chen, H. Tu, A. Zhang, Plasmonics 5, 221 (2010).

[21] Y. Cerenius, K. Ståhl, L.A. Svensson, T. Ursby, Å. Oskarsson, J. Albertsson, A. Liljas, J. Synch. Rad. 7, 203 (2000)

[22] M. Knaapila, C. Svensson, J. Barauskas, M. Zackrisson, S.S. Nielsen, N. Nørgaard Toft, B. Vestergaard, L. Arleth, Y. Cerenius, J. Synch. Rad. 16, 498 (2009).

[23] T.C. Huang, H. Toraya, T.N. Blanton, Y. Wu, J. Appl. Cryst. 26, 180 (1993).

[24] A.V. Semenyuk, D.I. Svergun, J. Appl. Crystallogr. 24, 537 (1991).

[25] S.R. Grobmyer, B.M. Moudgil, Cancer nanotechnology: methods and protocols, Humana Press, New York 2010.

[26] A. Guinier, Ann. Phys. 12, 166 (1939).

[27] M.S. Bakshi, P. Sharma, T.S. Banipal, Materials Lett. 61, 5004 (2007). 